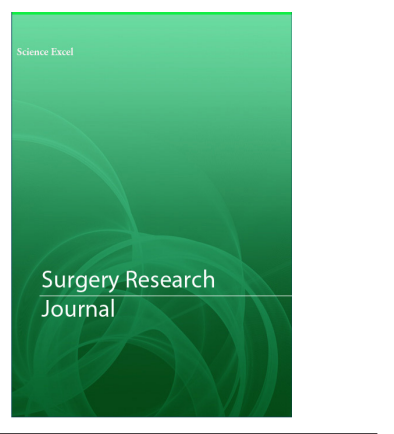

Correspondence

Devansh Saini

Industrial Engineering and Operations Research, Department of Mechanical and Industrial Engineering, University of Illinois, Chicago, USA.

E-mail:dsaini3@uic.edu

Tel: +1 (312) 383- 9221

- Received Date: 28 Jun 2021

- Accepted Date: 06 Jul 2021

- Publication Date: 12 Jul 2021

Copyright

(c) 2021 Science Excel. This is an openaccess article distributed under the terms of the Creative Commons Attribution 4.0 International license.

\title{
A systematic review of the latest technologies in Cranial Vault Remodeling and its outcomes for correction of craniosynostosis
}

\author{
Devansh Saini ${ }^{1 *}$, Quintin L Williams Jr. ${ }^{2}$, Lee Alkureishi ${ }^{3}$, Pravin Patel ${ }^{4}$, Linping \\ Zhao $^{5}$, Prashant Banerjee ${ }^{6}$, Jida Huang ${ }^{7}$ and Mathew Mathew ${ }^{8}$ \\ ${ }^{1} \mathrm{PhDc}$, Industrial Engineering and Operations Research, Department of Mechanical and Industrial Engineering, University of \\ Illinois, Chicago, USA
}

${ }^{2}$ Clinical Assistant Professor, Department of Mechanical and Industrial Engineering, University of Illinois, Chicago, USA

${ }^{3}$ Assistant Professor, Plastic, Reconstructive and Cosmetic Surgery, The Craniofacial Center, University of Illinois, Chicago, USA

${ }^{4}$ Professor, Plastic, Reconstructive and Cosmetic Surgery, The Craniofacial Center, University of Illinois, Chicago, USA

${ }^{5}$ Research Assistant Professor, Plastic, Reconstructive and Cosmetic Surgery, The Craniofacial Center, University of Illinois,

Chicago, USA

${ }^{6}$ Professor Emeritus, Department of Mechanical and Industrial Engineering, University of Illinois, Chicago, USA

${ }^{7}$ Assistant Professor, Department of Mechanical and Industrial Engineering, University of Illinois, Chicago, USA

${ }^{8}$ Associate Professor, Department of Bioengineering, University of Illinois, Chicago, USA

\begin{abstract}
Plastic surgeons often come across the dilemma of keeping operative time under check and achieving the "perfect" shape while still compassing the desired functional and aesthetic outcomes on the patient. This leads to a tradeoff between speed and accuracy in the operating room. Craniosynostosis (CS) is a congenital disability that is caused when one or more sutures of an infant's skull fuses too early before the brain is fully formed. This premature fusion of cranial sutures leads to abnormal head skull shape, increased intracranial pressure (ICP), and neurodevelopmental impairment for the child in later stages of life. Total Cranial Vault Remodeling (CVR) is a surgical procedure to treat this condition by reshaping the cranial bones, thus imparting a normative head shape for a child's brain to grow. These surgeries are usually lengthy and require the patient to be under prolonged anesthetic exposure and blood transfusion. Moreover, CVR is highly dependent on the surgeon's experience and lacks repeatability even though each case is specific and may require volatile manipulations that can often last hours. Based on our research question, we deployed the PRISMA protocol, exhausting databases to find relevant literature on this topic. The search strategy yielded a total of 399 research papers and after carefully reviewing each study, 12 papers were included in the systematic review to better understand the current engineering technologies being used effectively to improve the surgical outcomes for the correction of CS. Computer-aided design and manufacturing (CAD/CAM), Virtual Surgery Planning (VSP), threedimensional (3D) printing/CNC milling, resorbable plating system, 3D photogrammetry, 3D simulation, and intraoperative navigation are several technologies that can result in improving surgical outcomes for CVR surgery. Thus, there is a need to develop a workflow for the surgical treatment of CS using the latest technology that can potentially help surgeons achieve predictable and reproducible results in the operating room and be considered a standard across the craniofacial surgical community. A standardized workflow may also result in improving the speed (operative time) by moving part of the surgery to a more controlled pre-surgical planning environment and accuracy (precision) by achieving intricate and precise cuts on the calvarial bone, resulting in fewer errors during the surgery.
\end{abstract}

\section{Introduction}

The cranial vault, also known as calvaria, is the space within the skull that houses the brain. Its primary function is to protect the brain together with the base of the skull. Most characteristics of the human skull are formed during the initial two years of life and reach its conclusive size around the twentieth year of life. Craniosynostosis (CS) is a congenital condition that occurs in approximately one in 2000 to 2500 births [1]. Craniosynostosis, or simply synostosis, is the fusion of two or more bones of the skull. According to the literature, CS can be caused by various biomechanical, environmental, hormonal, and genetic factors with the number varying between syndromic and non-syndromic craniosynostosis. Most genetically determined CS is characterized by autosomal dominant inheritance, but around half of cases are accounted for by new mutations [2]. Genes most commonly mutated in CS are FGFR2, FGFR3, TWIST1, EFNB1, TCF12 and ERF, although mutations in just these six genes constitute three- 
quarters of all genetically diagnosed cases, the etiology of the remaining quarter is very diverse [3]. Syndromeassociated synostosis patients especially older infants having multisuture involvement are often presented with more severe deformity which cannot be adequately corrected by minimal access techniques [4]. Craniosynostosis is usually diagnosed during the birth of the infant or sometimes later when the family of the child suspects abnormal head shape. Doctors can confirm this via physical exam or medical imaging like CT/CAT scan, X-ray, and magnetic resonance imaging (MRI) which can provide the outline of the skull and conclusive evidence if the sutures have actually closed before their respective time.

According to the history, in 1851, Virchow first introduced the term 'craniosynostosis' and formulated what is today known as Virchow's law: When premature suture closure occurs, growth of the skull typically is restricted perpendicularly to the fused suture and enhanced in a plane parallel to it, thus trying to provide space for the fastgrowing brain [5]. The first reported surgical procedure for correction of CS was performed in 1890 by Lannelongue who advocated releasing, but not resecting, the fused suture [6]. Lannelongue in Paris described bilateral strip craniectomies for the treatment of CS, and Lane followed the intervention two years later in the United States. For the next 80 years, many new surgical techniques were devised and evaluated for their effectiveness in terms of reducing blood loss, patient health and overall complexity of the surgery. It was not until late 1960s, Tessier came along and introduced the procedure of Cranial Vault Remodeling (CVR) and new surgical protocols using instruments and power tools that were commonly available at that time and with the onset of CVR, strip craniectomy lost its popularity. Contrary to the advancements that took place in the field of science and medicine, these techniques have undergone relatively minor refinements over the last 60 years [7], and the overall method and tools (Figure 1) remains unchanged since Tessier showcased his technique at the International Meeting of Plastic Surgery in 1967.

In a typical CVR, the bones of the calvarium are carefully removed using a combination of a powered drill/burr and a high-speed rotary saw. Following removal, the shape of the bony segments is altered by sawing, bending, breaking, rotating or transposing the pieces akin to a jigsaw puzzle. They can then be fixed in their new relative positions with plates and screws, according to the surgeon's judgement.
The reconstructed "normative" cranial vault is achieved through trial and error until the satisfaction of the surgeon, and is often a compromise between achieving the "perfect" shape and keeping operative time to a minimum. There can be large bone gaps, flattened areas and/or sharp prominences between segments, which require significant bony remodeling to take place postoperatively. The outcome is often highly dependent upon the craftsmanship of the surgeon and complexity of the case. Though this procedure has become safer in the past few decades with significant reduction in morbidity and mortality rates [8-11], most craniofacial centers routinely admit patients to the intentsive care unit (ICU) after major cranial vault procedures [12]. Importantly, these are longer and potentially more bloody surgeries, with prolonged exposure to anesthetic agents and frequent need for blood transfusion in the infant population [13]. It has recently been suggested that there may be an association between early prolonged exposure to general anesthesia and neurodevelopmental deficits in later life [14]. This situation may be exacerbated for younger less experienced surgeons, where the operative time can be significantly longer compared with experienced surgeons [15]. In summary, reported blood loss, operative time, blood transfusion, hospital stay, and complications in later stages of life associated with these procedures can be significant and are well described throughout the craniofacial literature [16-20].

However, in the field of industrial engineering and manufacturing, the synergy between Computer-aided design and manufacturing (CAD/CAM), additive manufacturing (AM)/three dimensional (3D) printing, and computer-numerically-controlled $(\mathrm{CNC})$ machine tools have substantially replaced the use of manual/handheld tools, producing rapid improvements with efficiency, safety and accuracy. In the orthognathic surgical field, it has been shown that the use of computer-aided presurgical planning (referred as VSP) can provide multiple advantages including better visualization of the abnormality in 3D space, more precise orthognathic movements, and shorter operative times [21]. For this purpose, the current study was carried out with the primary goal of answering the following research question (RQ) based on our evaluation of the current situation:

$R Q$ - Are there certain engineering technologies mentioned in the existing literature that will result in advancing $C V R$ surgery to be more accurate, predictable and repeatable so that it would directly translate in improving surgical outcomes?

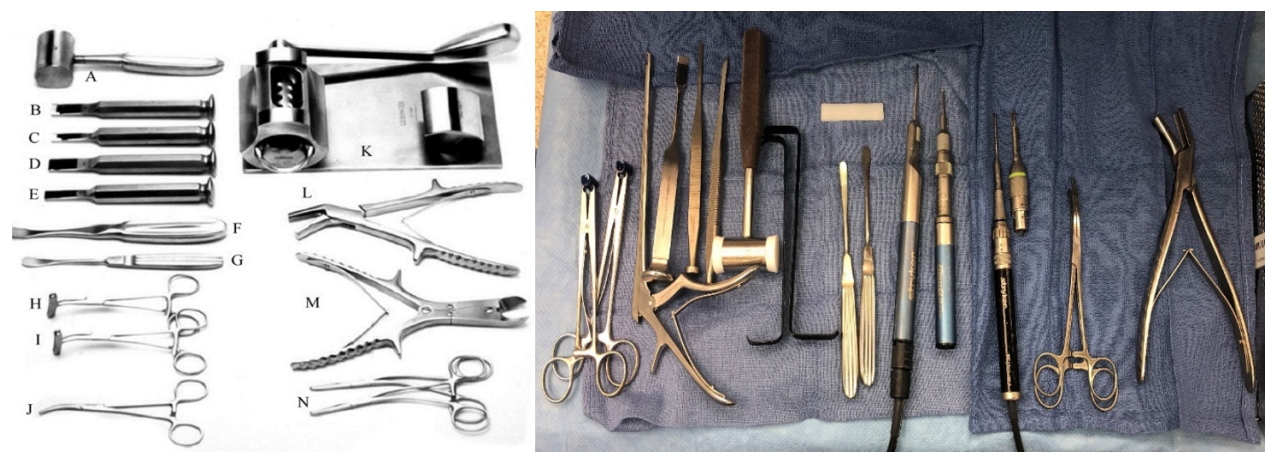

Figure 1. Comparison of tools presented (left) in A Man from Héric: The Life and Work of Paul Tessier, MD, Father of Craniofacial Surgery. Figure 27.1. (Copyright S. Anthony Wolfe), with the tools used (right) by craniofacial surgeons for a sagittal craniosynostosis case in 2020 at UI Hospital (Image courtesy of Lee Alkureishi MD). 
We hypothesize that the operative time and precision of surgery are the major surgical outcomes that can decrease the morbidity and mortality of the patient. Technologies like CAD/CAM along with 3D Printing/CNC Milling and VSP can help in improving the speed (operative time) by moving part of the surgery to a more controlled presurgical planning environment and accuracy (precision) by achieving intricate and precise cuts on the calvarial bone, resulting in fewer errors during the surgery. Inexperienced surgeons who start performing these types of surgeries will be able to go through a much reasonable learning curve due to the ability to move a large part of the surgery into presurgical planning setup, which gives surgeon confidence and capability to better visualize and rehearse the treatment and address any issues that were not apparent during initial examination. This reduction in learning curve of the surgeon over time will gradually bring down the experience curve of the surgery to standardize the protocol across the craniomaxillofacial surgical community. Additionally, the outcomes of VSP can be shared with patients' family, leading to an increased level of understanding and trust between the physician and the parents.

\section{Method}

We adopted the PRISMA search methodology [17] which stands for "Preferred Reporting Items for Systematic Reviews and Meta-Analyses", used to guide the process of literature review through described phases. It consists of four major phases starting from identification, which involves gathering of all the necessary article library through extensive database searching. The second step is the screening where the articles are assessed based on their abstracts and other metadata. Its followed by the eligibility phase where chosen articles are analyzed based on fulltext and inclusion criterion. Final step is inclusion, which involves showcasing the final set of included articles that are considered pivotal for the study and will be reviewed in detail.

We applied the PRISMA methodology in order to narrow down the articles needed to be reviewed that contain relevant details while ensuring the variety of research settings and outlooks towards the research question.

\section{Inclusion and exclusion criteria}

We included articles published within the last 10 years (2011-2020) to make sure that the current findings are based on the surgical techniques and tools that have been updated to the best of available technology, equipment and resources. This is also in parallel to the pivotal technologies like 3D printing/ AM, VSP and Virtual Reality (VR)/Augmented Reality (AR), that have been in the market for years but have only been commercialized in the past decade or so, especially for medical and surgical purposes. For article reliability, they had to be published in a peer-reviewed scholarly journal having a high Journal Impact Factor (JIF), in most cases a JIF of 2.0 or more. Grey literature composed of conference papers, patents, government reports, dissertation/thesis and other such material was excluded to strengthen the credibility of the review. Also, the language of the articles had to be English. Keywords that were used for literature search included "Craniosynostosis", "Cranial Vault Remodeling", and/or "Surgical Outcomes".

In terms of inclusion criteria, certain study parameters involving the surgical correction and its outcome had to be kept constant.

- Population: Mean age of 0-2 years for patients that were treated for CS was considered for the review since patient age of less than two years is considered optimal for the treatment using CVR

- Outcomes: Measurable outcomes were accepted over ambiguous ones since the later cannot be quantified

- Technology: Studies were required to suggest commercially available engineering technologies to be deemed pertinent in order to be a viable option for their inclusion in the systematic review

\section{Information sources}

Following databases were used for the study : (1) ScienceDirect, a platform for a wide variety of journals and e-books across all fields of natural sciences, applied sciences and technology, and social sciences, (2) PubMed, a service of the National Library of Medicine, that provides access to MEDLINE citations back to the mid-1960's to present, and (3) Web of Science, and their core collection which is the largest citation database in science, engineering, medicine and technology; also the social sciences, arts and humanities are represented.

\section{Results}

The search strategy used yielded a total of 399 research papers with ScienceDirect generating 184; PubMed, 148 and Web of Science, 67. A sample division of papers based on their respective field of science for one of the databases (Web of Science) is shown in Figure 2 below.

There were 33 duplicates that were found out amongst the databases which were later removed, leaving the final number to 366 scientific papers being included for screening. After careful examination of abstracts, 320 articles were excluded due to the reason codes (Table 1), leaving 46 articles for the full-text (FT) analysis. FT analysis was carried out to find if the articles were pertaining to the information that was consistent with the RQ under consideration. Further 29

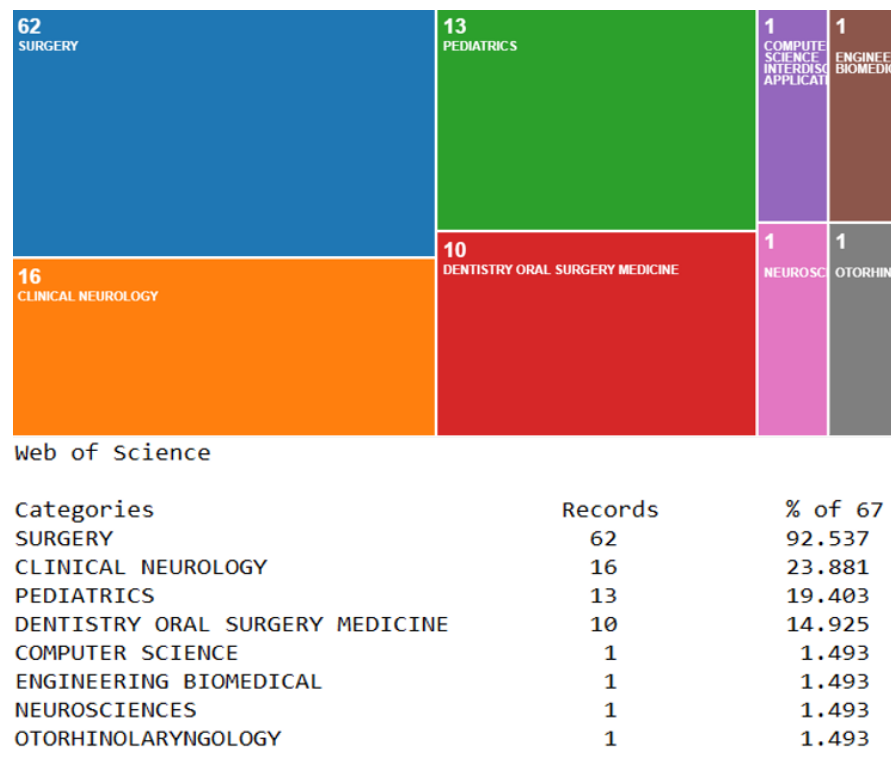

Figure 2. Categorical division of research articles for Web of Science 
Table 1. Reasons and their respective codes used in systematic review

\begin{tabular}{|c|c|}
\hline Reason for Exclusion & Code used \\
\hline Wrong study type & stu \\
\hline Wrong population & pop \\
\hline Wrong condition or surgical procedure & pro \\
\hline Wrong research focus & res \\
\hline Not improving surgerical procedure & sur \\
\hline Non-commercial technology used & tech \\
\hline Non measurable outcomes & out \\
\hline
\end{tabular}
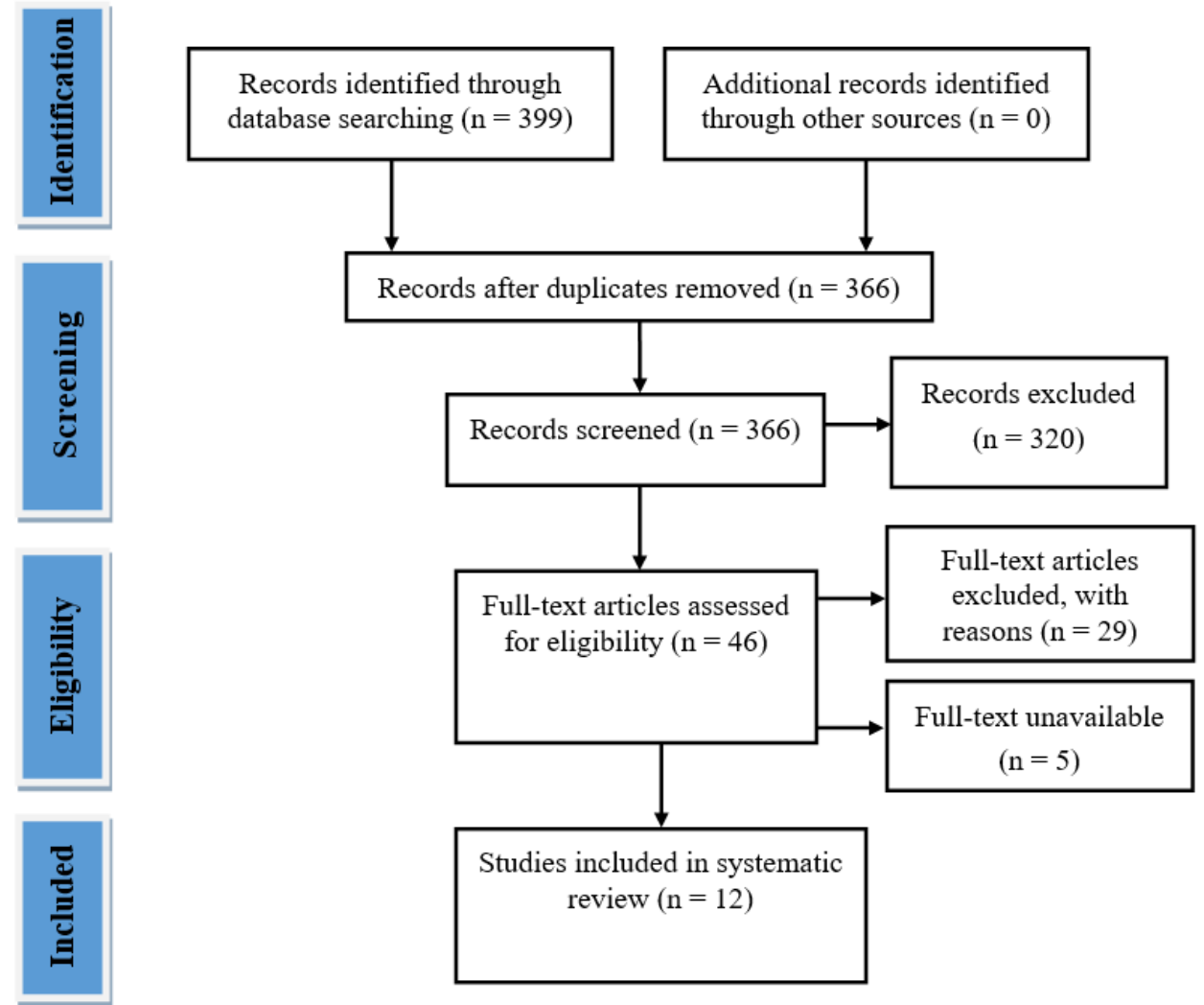

Figure 3. PRISMA flow diagram for systematic review

articles were lost to the reason codes used throughout the study and an additional five were not considered as their FT was unavailable, yielding a total of 12 papers to be included in this systematic review to better understand current engineering technologies being used effectively to improve the surgical outcomes for the correction of CS (Figure 3).

\section{Study characteristics}

The key characteristics of the study are presented in Table 2. All the included studies [23-34] were from past 10 years to align with the latest technology present in the market. The oldest study [28] was from 2012 and earliest [31] published in 2021. Sample size for patient population was diversified across the studies; three studies [27,30,31] had very small sample size $(n \leq 10)$, eight studies [23-25,28,29,32-34] had moderate sample size (between 10 to 75 ), while one study [26] $(n=203)$ had the largest patient population. Two studies $[25,29]$ mentioned median age while the rest of the studies $[23,24,26-28,30-34]$ provided mean age of the patients at the time of the surgery, which was considered to be less than two years for the review due to its efficacy for CVR. Several studies had an overlap in the technologies used which consisted of CAD/CAM, VSP, 3D printing, 3D simulation, resorbable plating, 3D photogrammetry, and Image Guided Surgery (IGS). Technologies were deployed at three categorically different stages; eight studies [23,27,28,30-34] used at pre-surgical stage, eight [24-30,32] during intraoperative and two $[28,34]$ at post-surgery stage either as a stand alone or in combination with each other. None of the technologies were in prototype stage and are commercially available at the time of the systematic review. Quantitative surgical outcomes were given preference to quantify the improvements after the technology was introduced.

Study results

The study results are summarized in table 3.

$\mathrm{Li}$, et al. [23] used computer-assisted 3D simulation combined with biomechanical calculations to decide the 
Table 2. Study characteristics

\begin{tabular}{|c|c|c|c|c|c|}
\hline $\begin{array}{l}\text { First Author } \\
\text { (Year) }\end{array}$ & Title & Population & Technology Used & $\begin{array}{l}\text { Deployment } \\
\text { Stage }\end{array}$ & Measured Surgical Outcomes \\
\hline $\begin{array}{l}\text { Xiang Li } \\
(2017)\end{array}$ & $\begin{array}{l}\text { Application of computer } \\
\text { assisted three-dimensional } \\
\text { simulation operation and } \\
\text { biomechanics analysis in } \\
\text { the treatment of sagittal } \\
\text { craniosynostosis }\end{array}$ & $\begin{array}{l}\mathrm{n}=18(11 \text { male and } 7 \\
\text { female }), \text { mean age }= \\
10.0 \pm 1.8 \text { months }\end{array}$ & $\begin{array}{l}\text { Computer-assisted } \\
\text { 3D simulation with } \\
\text { biomechanical analysis }\end{array}$ & pre & $\begin{array}{l}\text { Operative time, blood loss, } \\
\text { operation cost, Cranial index } \\
\text { (CI), Cranial circumference }(\mathrm{CC}) \text {, } \\
\text { Cranial vault asymmetry index } \\
\text { (CVAI), and postoperative } \\
\text { complications }\end{array}$ \\
\hline $\begin{array}{l}\text { Christian } \\
\text { Freudlsperger } \\
(2014)\end{array}$ & $\begin{array}{l}\text { The value of ultrasound- } \\
\text { assisted pinned resorbable } \\
\text { osteosynthesis for cranial vault } \\
\text { remodelling in craniosynostosis }\end{array}$ & $\begin{array}{l}\mathrm{n}=38, \text { mean age }= \\
11.3 \text { months }\end{array}$ & $\begin{array}{l}\text { Ultrasound-assisted pinned } \\
\text { resorbable osteosynthesis }\end{array}$ & intra & $\begin{array}{l}\text { Operation time, stability of the } \\
\text { surgical result, local infection rate, } \\
\text { and palpability or visibility of the } \\
\text { plates. }\end{array}$ \\
\hline $\begin{array}{l}\text { Niina } \\
\text { Salokorpi } \\
(2015)\end{array}$ & $\begin{array}{l}\text { Frontal cranial modeling } \\
\text { using endocranial resorbable } \\
\text { plate fixation in } 27 \\
\text { consecutive plagiocephaly and } \\
\text { trigonocephaly patients }\end{array}$ & $\begin{array}{l}\mathrm{n}=27(15 \text { male and } \\
12 \text { female }), \text { median } \\
\text { age }=13.4 \text { months }\end{array}$ & Resorbable plating system & intra & $\begin{array}{l}\text { Operative time, bleeding, learning } \\
\text { curve. }\end{array}$ \\
\hline $\begin{array}{l}\text { Leslie G. } \\
\text { Branch (2017) }\end{array}$ & $\begin{array}{l}\text { Long-Term Outcomes of } \\
\text { Pediatric Cranial Reconstruction } \\
\text { Using Resorbable Plating } \\
\text { Systems for the Treatment of } \\
\text { Craniosynostosis }\end{array}$ & $\begin{array}{l}\mathrm{n}=203(115 \text { male and } \\
88 \text { female }), \text { mean age } \\
=15.8 \text { months }\end{array}$ & Resorbable plating system & intra & $\begin{array}{l}\text { Blood loss, transfusion rate, } \\
\text { transfused volume, length of } \\
\text { hospital stay, ICU stay, re- } \\
\text { operation rate }\end{array}$ \\
\hline $\begin{array}{l}\text { Samir Mardini } \\
(2014)\end{array}$ & $\begin{array}{l}\text { Three-dimensional preoperative } \\
\text { virtual planning and template } \\
\text { use for surgical correction of } \\
\text { craniosynostosis" }\end{array}$ & $\begin{array}{l}\mathrm{n}=4 \text { (all male) }, \text { mean } \\
\text { age }=10.75 \text { months }\end{array}$ & $\begin{array}{l}\text { Virtual surgery planning } \\
\text { (VSP), computer-assisted } \\
\text { design/computer- } \\
\text { assisted manufacturing } \\
\text { (CAD/CAM), CNC } \\
\text { Machining/3D Printing } \\
\text { (pre-fabricated template) }\end{array}$ & pre and intra & $\begin{array}{l}\text { Surgical time, blood loss, blood } \\
\text { transfusion, hospital length of stay }\end{array}$ \\
\hline $\begin{array}{l}\text { Jan-Falco } \\
\text { Wilbrand } \\
(2012)\end{array}$ & $\begin{array}{l}\text { Objectification of cranial vault } \\
\text { correction for craniosynostosis } \\
\text { by three-dimensional } \\
\text { photography }\end{array}$ & $\begin{array}{l}\mathrm{n}=28(19 \text { male and } 9 \\
\text { female }), \text { mean age }= \\
7.8 \text { months }\end{array}$ & 3D Photogrammetry & pre and post & $\begin{array}{l}\text { Cephalic Index }(\mathrm{CI}) \text {, anterior } \\
\text { symmetry ratio, posterior } \\
\text { symmetry ratio, anterior skull } \\
\text { volume }\end{array}$ \\
\hline $\begin{array}{l}\text { Rajiv R. Iyer } \\
(2018)\end{array}$ & $\begin{array}{l}\text { Use of computer-assisted design } \\
\text { and manufacturing to localize } \\
\text { dural venous sinuses during } \\
\text { reconstructive surgery for } \\
\text { craniosynostosis }\end{array}$ & $\begin{array}{l}\mathrm{n}=32(18 \text { male and } \\
14 \text { female }), \text { median } \\
\text { age }=11 \text { months }\end{array}$ & $\begin{array}{l}\text { Computer-aided } \\
\text { design/computer-aided } \\
\text { manufacturing }(\mathrm{CAD} / \\
\mathrm{CAM})\end{array}$ & intra & $\begin{array}{l}\text { Superior sagittal sinus (SSS) } \\
\text { deviation, operative duration, } \\
\text { blood loss, hospital length of stay }\end{array}$ \\
\hline $\begin{array}{l}\text { David García- } \\
\text { Mato (2019) }\end{array}$ & $\begin{array}{l}\text { Craniosynostosis surgery: } \\
\text { workflow based on virtual } \\
\text { surgical planning, intraoperative } \\
\text { navigation and 3D printed } \\
\text { patient-specific guides and } \\
\text { templates }\end{array}$ & $\begin{array}{l}\mathrm{n}=5(2 \text { male and } 3 \\
\text { female }), \text { mean age }= \\
11 \text { months }\end{array}$ & $\begin{array}{l}\text { Computer-aided } \\
\text { design/computer-aided } \\
\text { manufacturing (CAD/ } \\
\text { CAM), 3D Printing, Virtual } \\
\text { Surgical Planning (VSP), } \\
\text { Intraoperative navigation }\end{array}$ & pre and intra & $\begin{array}{l}\text { Root-mean-squared error (RMSE), } \\
\text { interfrontal angle, transverse } \\
\text { forehead width }\end{array}$ \\
\hline $\begin{array}{l}\text { Praveen } \\
\text { Ganesh (2021) }\end{array}$ & $\begin{array}{l}\text { Traditional Versus Virtual } \\
\text { Surgery Planning of the Fronto- } \\
\text { Orbital Unit in Anterior Cranial } \\
\text { Vault Remodeling Surgery }\end{array}$ & $\begin{array}{l}\mathrm{n}=10,5 \text { TFOU group } \\
\text { (mean age }=7.4 \\
\text { months) and } 5 \text { NFOU } \\
\text { group (mean age }=7 \\
\text { months) patients }\end{array}$ & $\begin{array}{l}\text { Computer-aided } \\
\text { design/computer-aided } \\
\text { manufacturing (CAD/ } \\
\text { CAM), Virtual Surgical } \\
\text { Planning (VSP), 3D } \\
\text { Printing }\end{array}$ & pre and intra & $\begin{array}{l}\text { Time of surgery, blood loss, length } \\
\text { of hospital stay }\end{array}$ \\
\hline $\begin{array}{l}\text { Tom W. } \\
\text { Andrew (2018) }\end{array}$ & $\begin{array}{l}\text { Virtual Surgical Planning } \\
\text { Decreases Operative Time } \\
\text { for Isolated Single Suture and } \\
\text { Multi-suture Craniosynostosis } \\
\text { Repair" }\end{array}$ & $\begin{array}{l}\mathrm{n}=63,35 \text { non-VSP } / \\
\text { control (mean age }= \\
27.23 \text { months) and } \\
28 \mathrm{VSP} \text { (mean age }= \\
19.75 \text { months) cases }\end{array}$ & $\begin{array}{l}\text { Virtual Surgical Planning } \\
\text { (VSP) }\end{array}$ & pre & $\begin{array}{l}\text { Number of screws, number of } \\
\text { plates, blood loss, hospital stay, } \\
\text { postoperative complications, } \\
\text { operative time }\end{array}$ \\
\hline $\begin{array}{l}\text { David Y. } \\
\text { Khechoyan } \\
(2014)\end{array}$ & $\begin{array}{l}\text { Surgical outcomes in } \\
\text { craniosynostosis reconstruction: } \\
\text { The use of prefabricated } \\
\text { templates in cranial vault } \\
\text { remodelling }\end{array}$ & $\begin{array}{l}\mathrm{n}=37(20 \text { male and } 17 \\
\text { female) } 14 \text { template } \\
\text { (mean age }=11.5 \\
\text { months) and } 23 \text { no } \\
\text { template/control } \\
\text { (mean age }=10.2 \\
\text { months) patients }\end{array}$ & $\begin{array}{l}\text { Computer-aided } \\
\text { design/manufacturing } \\
\text { (CAD/CAM), CNC } \\
\text { Machining/3D Printing } \\
\text { (pre-fabricated template) }\end{array}$ & pre and intra & $\begin{array}{l}\text { Area under the curve (AUC), } \\
\text { duration of operation, blood loss, } \\
\text { blood transfusion and length of } \\
\text { hospital stay. }\end{array}$ \\
\hline $\begin{array}{l}\text { Christian } \\
\text { Freudlsperger } \\
(2015)\end{array}$ & $\begin{array}{l}\text { Metopic synostosis: Measuring } \\
\text { intracranial volume change } \\
\text { following fronto-orbital } \\
\text { advancement using three- } \\
\text { dimensional photogrammetry }\end{array}$ & $\begin{array}{l}\mathrm{n}=18 \text { (all males }) \\
\text { mean age }=9 \text { months }\end{array}$ & 3D Photogrammetry & pre and post & $\begin{array}{l}\text { Intracranial volume (total, frontal } \\
\text { and posterior) }\end{array}$ \\
\hline
\end{tabular}


Table 3. Summary of study reports

\begin{tabular}{|c|c|c|}
\hline $\begin{array}{c}\text { First Author } \\
\text { (Year) }\end{array}$ & Title & Results \\
\hline $\begin{array}{l}\text { Xiang Li } \\
(2017)\end{array}$ & $\begin{array}{l}\text { Application of computer } \\
\text { assisted three-dimensional } \\
\text { simulation } \\
\text { operation and biomechanics } \\
\text { analysis in the treatment of } \\
\text { sagittal craniosynostosis }\end{array}$ & $\begin{array}{l}\text { Length of operation was }(276 \pm 23.5 \mathrm{~min}) \text {, blood loss was }(90 \pm 15.5 \mathrm{ml}) \text {, operation cost was } \\
(34,495 \pm 8662 ¥) \text {, pre operative CVAI was } 2.2 \pm 0.9 \% \text {, while the post-operative CVAI was } \\
3.6 \pm 0.6 \% \text { for conventional Method (Group A). Length of operation was }(217 \pm 29.3 \mathrm{~min}) \text {, } \\
\text { blood loss was }(70 \pm 11.7 \mathrm{ml}) \text {, operation cost was }(25,149 \pm 4133 ¥) \text {, pre-operative CVAI } \\
\text { was } 2.05 \pm 0.8 \% \text {, while the post-operative CVAI was } 2.1 \pm 0.6 \% \text { using proposed technology } \\
\text { (Group B). CI was significantly improved during post-operative period In group A (PCI(A) } \\
=0.005<0.05) \text {, as well as for group B in post-operative period (PCI }(\mathrm{B})=0.012<0.05) \text {. No } \\
\text { statistical difference between pre and post-operative CC in group A (Pcc }(\mathrm{A})=0.643<0.05) \text {, } \\
\text { as well as group B (Pcc }(\mathrm{B})=0.137<0.05) \text {. No postoperative complication was observed for } \\
\text { group B, while there was } 1 \text { case of central nervous system infection that occurred in group A. }\end{array}$ \\
\hline $\begin{array}{l}\text { Christian } \\
\text { Freudlsperger } \\
(2014)\end{array}$ & $\begin{array}{l}\text { The value of ultrasound- } \\
\text { assisted pinned resorbable } \\
\text { osteosynthesis for cranial } \\
\text { vault remodelling in } \\
\text { craniosynostosis }\end{array}$ & $\begin{array}{l}\text { Mean operation time in } 14 \text { cases of trigonocephaly }(152.5 \mathrm{~min}) \text { and in } 18 \text { cases of } \\
\text { scaphocephaly }(151.05 \mathrm{~min}) \text { was not significantly longer than older collective of } 50 \text { patients } \\
\text { with metopic synostosis and } 96 \text { patients with sagittal synostosis where conventional titanium } \\
\text { plates had been used }(\mathrm{p}<0.05) \text {. Wound healing was uneventful during the inpatient period } \\
\text { of } 10-14 \text { days. During the follow-up examinations all remodelled cranial vaults were stable } \\
\text { on clinical palpation. There were no cases of palpable bone flap settling. Clinical signs of } \\
\text { inflammation of infection in the area of previous surgery were not seen in the complete } \\
\text { follow-up period, except for one patient with signs of inflammatory skin reaction after } 8 \\
\text { weeks, which recovered spontaneous after } 5 \text { days. No signs of foreign body reaction to the } \\
\text { resorbable material were observed. The number of palpable or visible plates, respectively, } \\
\text { increased during the first months with a maximum at } 12 \text { months; } 34(89 \%) \text { plates palpable } \\
\text { and } 26(68 \%) \text { plates visible. After this time point, the number of palpable and/or visible plates } \\
\text { decreased continuously until the at end of the follow-up period of } 21 \text { month; } 3(20 \%) \text { were } \\
\text { plates palpable and } 0(0 \%) \text { plates were visible. }\end{array}$ \\
\hline $\begin{array}{l}\text { Niina } \\
\text { Salokorpi } \\
(2015)\end{array}$ & $\begin{array}{l}\text { Frontal cranial modeling } \\
\text { using endocranial resorbable } \\
\text { plate fixation in } 27 \\
\text { consecutive plagiocephaly } \\
\text { and trigonocephaly patients }\end{array}$ & $\begin{array}{l}\text { The mean operative time was } 210 \mathrm{~min} \text { (range } 95-315 \mathrm{~min} \text {, SD } 54.3,95 \% \mathrm{CI}=188.9-231.8 \text { ). } \\
\text { The trigonocephaly group required less operative time (average } 170 \mathrm{~min} \text { ) than operations } \\
\text { for the plagiocephaly group (average } 234 \mathrm{~min} \text { ). Median bleeding was } 300 \mathrm{ml} \text { (SD } 328.1 \text {, } \\
95 \% \mathrm{CI}=260.2-519.8 \text {, range } 90-1700 \mathrm{ml} \text { ). There was no significant difference between the } \\
\text { two diagnostic groups with regards to intraoperative blood loss ( } \mathrm{p}=0.227 \text { ). To evaluate the } \\
\text { influence of learning curve on the length of operation, patients were divided in two groups: } 13 \\
\text { patients that were operated between } 1998 \text { and } 2007 \text { (mean operative time } 232 \mathrm{~min} \text {, SD 54.4) } \\
\text { and } 14 \text { patients that were operated } 2008 \text { and later (mean operative time } 190 \mathrm{~min} \text {, SD } 47.4 \text { ). } \\
\text { There was a statistically significant difference between these two groups (p=0.043). There was } \\
\text { a tendency for current surgeries to require an average of } 49 \text { min less operative time. }\end{array}$ \\
\hline $\begin{array}{l}\text { Leslie G. } \\
\text { Branch } \\
(2017)\end{array}$ & $\begin{array}{l}\text { "Long-Term Outcomes } \\
\text { of Pediatric Cranial } \\
\text { Reconstruction } \\
\text { Using Resorbable Plating } \\
\text { Systems for the Treatment } \\
\text { of Craniosynostosis" }\end{array}$ & $\begin{array}{l}\text { Mean estimated blood loss was } 93 \mathrm{~mL} \text { (range } 30-650 \mathrm{~mL} \text { ) and transfusion was required in } \\
48.3 \% \text { with mean transfused volume of } 223 \mathrm{~mL} \text { (range } 70-675 \mathrm{~mL} \text { ). Mean length of hospital } \\
\text { stay was } 5.4 \text { days (range } 3-14 \text { days). Mean ICU stay was } 1.3 \text { days (range } 1-8 \text { days). Overall, } \\
\text { reoperation due to complications was required in } 5.4 \% \text { of children. }\end{array}$ \\
\hline $\begin{array}{l}\text { Samir } \\
\text { Mardini } \\
(2014)\end{array}$ & $\begin{array}{l}\text { Three-dimensional } \\
\text { preoperative virtual } \\
\text { planning and template use } \\
\text { for surgical correction of } \\
\text { craniosynostosis }\end{array}$ & $\begin{array}{l}\text { The mean surgical time was } 326 \mathrm{~min} \text {, mean estimated blood loss was } 437.5 \mathrm{ml} \text {, mean blood } \\
\text { transfusion was } 329.25 \mathrm{ml} \text { and the mean hospital length of stay was } 4.5 \text { days. }\end{array}$ \\
\hline $\begin{array}{l}\text { Jan-Falco } \\
\text { Wilbrand } \\
(2012)\end{array}$ & $\begin{array}{l}\text { Objectification of cranial } \\
\text { vault correction for } \\
\text { craniosynostosis by three- } \\
\text { dimensional photography" }\end{array}$ & $\begin{array}{l}\text { The Cephalic Index in Scaphocephaly changed from } 75.1 \% \text { to a median value of } 77.4 \% \text {. The } \\
\text { anterior symmetry ratio for coronal synostoses improved from } 0.943 \text { to } 0.949 \text { (a value of } 1.0 \\
\text { represents perfect symmetry). The posterior symmetry ratio improved from } 0.733 \text { to } 0.808 \\
\text { postoperatively in one single lambdoidal synostosis. In trigonocephaly, the median anterior } \\
\text { skull volume rose from } 528 \text { to } 601 \mathrm{ml} \text {. }\end{array}$ \\
\hline $\begin{array}{l}\text { Rajiv R. Iyer } \\
(2018)\end{array}$ & $\begin{array}{l}\text { Use of computer-assisted } \\
\text { design and manufacturing } \\
\text { to localize dural venous } \\
\text { sinuses during reconstructive } \\
\text { surgery for craniosynostosis }\end{array}$ & $\begin{array}{l}\text { Mean ( } \pm \text { standard deviation) operative duration was } 8.4 \mathrm{~h}( \pm 1.3) \text {. Median estimated blood } \\
\text { loss was } 350 \mathrm{~mL} \text {. Median hospital length of stay was } 3.5 \text { days. All patients tolerated surgical } \\
\text { intervention without significant complications. Sagittal sinus deviation from the sagittal suture } \\
\text { was maximal in unicoronal synostosis patients }(10.2 \pm 0.9 \mathrm{~mm}) \text {. }\end{array}$ \\
\hline
\end{tabular}




\begin{tabular}{|c|c|c|}
\hline $\begin{array}{l}\text { First Author } \\
\text { (Year) }\end{array}$ & Title & Results \\
\hline $\begin{array}{l}\text { David } \\
\text { García-Mato } \\
(2019)\end{array}$ & $\begin{array}{l}\text { Craniosynostosis surgery: } \\
\text { workflow based on } \\
\text { virtual surgical planning, } \\
\text { intraoperative navigation and } \\
\text { 3D printed patient-specific } \\
\text { guides and templates }\end{array}$ & $\begin{array}{l}\text { Primary registration, which was performed using six landmarks on the 3D printed surgical } \\
\text { guides, yielded an average RMSE of } 0.94 \pm 0.27 \mathrm{~mm} \text { for the five surgeries under study. } \\
\text { The average time required to perform the registrations was } 50 \pm 10 \text { seconds. Secondary } \\
\text { registration, which was performed using the position of six resorbable pins attached } \\
\text { intraoperatively, yielded an average RMSE of } 1.30 \pm 0.47 \mathrm{~mm} \text {. The average duration } \\
\text { of secondary registrations was } 40 \pm 14 \text { seconds. The average error between target and } \\
\text { postoperative interfrontal angles was } 3.63^{\circ} \text {. The average error between target and transverse } \\
\text { forehead width was } 1.89 \mathrm{~mm} \text {. }\end{array}$ \\
\hline $\begin{array}{l}\text { Praveen } \\
\text { Ganesh } \\
(2021)\end{array}$ & $\begin{array}{l}\text { Traditional Versus Virtual } \\
\text { Surgery Planning of the } \\
\text { Fronto-Orbital Unit in } \\
\text { Anterior Cranial Vault } \\
\text { Remodeling Surgery }\end{array}$ & $\begin{array}{l}\text { The Mean }( \pm \mathrm{SD}) \text { time of surgery was } 364 \pm 32.09 \text { minutes in NFOU group when compared to } \\
\text { the VFOU group where the Mean }( \pm \mathrm{SD}) \text { time of surgery was } 310 \pm 29.15 \text { minutes. The Time } \\
\text { of Surgery was significantly low in the VFOU group than the NFOU Group with P }=0.021 \\
(<0.05) \text {. Intra-operative mean blood loss was found to be } 250 \pm 23.45 \mathrm{ml} \text { in NFOU group } \\
\text { compared to } 218 \pm 13.03 \mathrm{ml} \text { in the VFOU group. There was a statistically significant difference } \\
\text { in p Value }=0.014(<0.05) \text { between the groups. There was no statistically significant } \\
\text { difference in the length of hospital stay with an average of } 5 \text { days in both the groups. }\end{array}$ \\
\hline $\begin{array}{l}\text { Tom W. } \\
\text { Andrew } \\
(2018)\end{array}$ & $\begin{array}{l}\text { Virtual Surgical Planning } \\
\text { Decreases Operative } \\
\text { Time for Isolated Single } \\
\text { Suture and Multi-suture } \\
\text { Craniosynostosis Repair }\end{array}$ & $\begin{array}{l}100 \text { screws or rivets were used in the VSP group in comparison to } 66 \text { screws (no rivets) in } \\
\text { the surgical controls }(\mathrm{P}<0.01) \text {. group. No difference in the number of plates opened from } \\
\text { original packaging was found }(4.89 \text {, controls and } 4.39 \text {, VSP). The Intraoperative blood } \\
\text { loss was comparable in both groups, } 172.21 \mathrm{ml} \text { and } 182.86 \mathrm{ml} \text { for controls and VSP cases, } \\
\text { respectively. VSP cases stayed in hospital significantly longer than control patients, } 5.39 \text { days } \\
\text { versus } 3.97 \text { days }(\mathrm{P}<0.01) \text {, respectively. Postoperative complications were similar in both } \\
\text { groups. The operative time for all procedures was on average } 321.44 \text { minutes in the control } \\
\text { group and } 265.61 \text { in the VSP cases }(\mathrm{P}=0.01) \text {. }\end{array}$ \\
\hline $\begin{array}{l}\text { David Y. } \\
\text { Khechoyan } \\
(2014)\end{array}$ & $\begin{array}{l}\text { Surgical outcomes in } \\
\text { craniosynostosis } \\
\text { reconstruction: The use of } \\
\text { prefabricated } \\
\text { templates in cranial vault } \\
\text { remodelling }\end{array}$ & $\begin{array}{l}\text { Estimated blood loss, volume of blood product transfusion and length of hospital stay was } \\
\text { not statistically different between the two groups. The duration of operation was significantly } \\
\text { shorter for the template group }(212 \mathrm{vs} .258 \mathrm{~min}, \mathrm{p}<0.001) \text {. At the postoperative time point, } \\
\text { the mean AUC for the template and the no-template groups was significantly different ( } 199 \\
\left.\text { vs. } 309 \mathrm{~mm}^{2}, \mathrm{p}<0.05\right) \text {. Mean reduction in AUC at the time of operation was } 74 \% \text { for the } \\
\text { template group and } 56 \% \text { for the no-template (control) group, respectively }(\mathrm{p}<0.05) \text {. The } \\
\text { mean absolute reduction in AUC was also significantly different between the template and no- } \\
\left.\text { template, control groups ( } 663 \text { vs. } 500 \mathrm{~mm}^{2}, \mathrm{p}<0.001\right) \text {. }\end{array}$ \\
\hline $\begin{array}{l}\text { Christian } \\
\text { Freudlsperger } \\
(2015)\end{array}$ & $\begin{array}{l}\text { Metopic synostosis: } \\
\text { Measuring intracranial } \\
\text { volume change following } \\
\text { fronto-orbital advancement } \\
\text { using three-dimensional } \\
\text { photogrammetry }\end{array}$ & $\begin{array}{l}\text { The measurement of the mean pre- and post-operative intracranial volume revealed a } \\
\text { significant increase of the frontal volume }(707.5 \mathrm{ml} \text { vs. } 833.3 \mathrm{ml})(\mathrm{p}=0.0054) \text { after fronto- } \\
\text { orbital advancement. The mean posterior volume's increase was less pronounced }(654.5 \mathrm{ml} \\
\text { vs. } 686.0 \mathrm{ml})(\mathrm{p}=0.3376) \text {. The operative procedure increased the mean total volume }(1362.0 \\
\mathrm{ml} \text { vs. } 1519.3 \mathrm{ml}) \text { in a significant manner }(\mathrm{p}<0.01) \text {. Pre-operatively, the mean frontal volume } \\
\text { of children with metopic synostosis was significantly lower compared with the control group } \\
(654.5 \mathrm{ml} \text { vs. } 911.0 \mathrm{ml})(\mathrm{p}<0.001) \text {. Consequently, mean total volume was significantly } \\
\text { decreased }(1362.0 \mathrm{ml} \mathrm{vs} .1541 .8 \mathrm{ml})(\mathrm{p}=0.0031) \text {. Posterior volume did not differ from the } \\
\text { control group in a significant manner }(654.5 \mathrm{ml} \text { vs. } 630.8 \mathrm{ml})(\mathrm{p}=0.4322) \text {. Post-operatively, } \\
\text { no significant difference in the frontal, posterior and total volume was present, indicating } \\
\text { a considerable increase in the intracranial volume especially in the frontal area due to the } \\
\text { fronto-orbital advancement. }\end{array}$ \\
\hline
\end{tabular}

position and number of fixture for treatment of CS. Eighteen patients were split into two groups as 10 patients were in Group A (traditional method) and 8 patients in Group B (new treatment method). Patient mean age was $10.0 \pm 1.8$ months, consisting of 11 males and 7 females. Cranial index (CI), Cranial circumference (CC), and Cranial vault asymmetry index (CVAI) were measured along with operative time, blood loss, operation cost, and postoperative complications. A virtual head model was established by loading CT data into Mimics software (Mimics 17.0, Materialize NV) which was used to complete an individualized and optimal program for the patients while measuring CI, CC, and CVAI. Finished reconstruction head model was further imported to CATIA software (CATIA V5) to obtain "igs" format model before finally using Hypermesh software to set bone and plating material properties to conduct biomechanical testing. Using a 3D finite element model (FEM), force distribution was evaluated to guide placement and removal of additional fixation material. Optimal location and amount of fixation was finalized after multiple iterations of the process. For Group A (conventional method), length of operation was (276 $\pm 23.5 \mathrm{~min})$, blood loss was $(90 \pm 15.5 \mathrm{ml})$, operation cost was $(34,495 \pm 8662 ¥)$, pre-operative CVAI was $2.2 \pm$ $0.9 \%$, while the post-operative CVAI was $3.6 \pm 0.6 \%$. While for Group B (proposed technology), length of operation was (217 $\pm 29.3 \mathrm{~min})$, blood loss was $(70 \pm 11.7 \mathrm{ml})$, operation cost was $(25,149 \pm 4133 ¥)$, pre-operative CVAI was $2.05 \pm 0.8 \%$, while the post-operative CVAI was $2.1 \pm 0.6 \%$. Cranial index was significantly improved during post-operative period in Group A $(\mathrm{PCI}(\mathrm{A})=0.005<0.05)$, as well as for Group B in post-operative period $(\mathrm{PCI}(\mathrm{B})=0.012<0.05)$. No statistical difference was observed between pre and post-operative 
CC in Group A $(\operatorname{Pcc}(A)=0.643<0.05)$, as well as Group B $(\operatorname{Pcc}(B)=0.137<0.05)$. No postoperative complication was observed for Group B, while there was one case of central nervous system infection that occurred in Group A.

Freudlsperger, et al. [24] focused on ultrasound-assisted pinned resorbable system developed to provide a timesaving and stable osteosynthesis for CS correction. Thirty eight patients were part of the study with mean age of 11.3 months. Operation time, stability of the surgical result, local infection rate, and palpability or visibility of the plates was evaluated in the study. In this technology, PDLLA [poly D-lactide (50\%) and L-lactide acid (50\%)] pins are inserted after conventional hole drilling with the aid of ultrasound to weld with the bone in order to fix plate or mesh. A standard protocol was followed to place plates and pins on the patients depending upon the type of synostosis. A minimum of 15 and a maximum of 21 months was considered for follow-up. Mean operation time in 14 cases of trigonocephaly (152.5 $\mathrm{min}$ ) and in 18 cases of scaphocephaly (151.05 min) was not significantly longer than older collective of 50 patients with metopic synostosis and 96 patients with sagittal synostosis where conventional titanium plates had been used $(\mathrm{p}<$ 0.05). Wound healing was uneventful during the inpatient period of 10-14 days. During the follow-up examinations all remodeled cranial vaults were stable on clinical palpation. There were no cases of palpable bone flap settling. Clinical signs of inflammation of infection in the area of previous surgery were not seen in the complete follow-up period, except for one patient with signs of inflammatory skin reaction after 8 weeks, which recovered spontaneous after 5 days. No signs of foreign body reaction to the resorbable material were observed. The number of palpable or visible plates, respectively, increased during the first months with a maximum at 12 months; $34(89 \%)$ plates palpable and 26 $(68 \%)$ plates visible. After this time point, the number of palpable and/or visible plates decreased continuously until at end of the follow-up period of 21 month; 3 (20\%) were plates palpable and $0(0 \%)$ plates were visible.

Salokorpi, et al. [25] evaluated endocranial resorbable plate fixation made up of poly (lactide-co-glcolide (PLGA) and its clinical and radiographic results on synostosis patients. Twenty seven patients participated in the study having a median age of 13.4 months in which 15 were male and 12 were female. Though aesthetic outcome was considered the primary outcome of the study, it was not considered for the purposes of review as it is a subjective outcome which cannot be quantified. Instead, operative time, bleeding, and learning curve were examined. The mean operative time was $210 \mathrm{~min}$ (range 95-315 min, standard deviation [SD] 54.3, $95 \%$ confidence interval $[\mathrm{CI}]=188.9-231.8)$. The PLGA plates were fixed on the inner surface of the dissected cranial bones, and the bone-plate construct was molded as symmetrical as possible. In 16 cases, the plates were fixed to the skull using screws (Inion CPS Baby, Tampere, Finland), whereas in rest of the 11 cases, fixation was performed using tacks from the Sonic Weld System (KLS Martin). The trigonocephaly group required less operative time (average $170 \mathrm{~min}$ ) than operations for the plagiocephaly group (average $234 \mathrm{~min})$. Median bleeding was $300 \mathrm{ml}$ (SD 328.1, $95 \%$ CI $=260.2-519.8$, range $90-1700 \mathrm{ml}$ ). There was no significant difference between the two diagnostic groups with regards to intraoperative blood loss $(p=0.227)$. To evaluate the influence of learning curve on the length of operation, patients were divided in two groups: 13 patients that were operated between 1998 and 2007 (mean operative time $232 \mathrm{~min}, \mathrm{SD} 54.4$ ) and 14 patients that were operated 2008 and later (mean operative time $190 \mathrm{~min}$, SD 47.4). There was a statistically significant difference between these two groups $(p=0.043)$. There was a tendency for current surgeries to require an average of $49 \mathrm{~min}$ less operative time.

Branch, et al. [26] used resorbable plating system composed of polyglycolic and polylactic acids from MacroPore (Cytori Therapeutics, San Diego, CA) or Lactosorb (Walter Lorenz Surgical Inc, Jacksonville, FL) for treatment of CS. A total of 203 patients (115 male and 88 female) were part of the study with a mean age of 15.8 months. Blood loss, transfusion rate, transfused volume, length of hospital stay, ICU stay, and re-operation rate were analyzed in this study. During surgery, initial pilot predrilling was done for placing plates and screws, followed by hand tapping and placement of threaded screw. Mean estimated blood loss was $93 \mathrm{~mL}$ (range 30-650 $\mathrm{mL}$ ) and blood transfusion was required in $48.3 \%$ with mean transfused volume of $223 \mathrm{~mL}$ (range 70-675 mL). Mean length of hospital stay was 5.4 days (range 3-14 days), while mean ICU stay was 1.3 days (range 1-8 days). Overall, reoperation due to complications was required in $5.4 \%$ of children.

Mardini, et al. [27] investigated the use of VSP, CAD/ CAM, and pre-fabricated template for the correction of CS. The study was limited to only 4 patients that were all male having a mean age of 10.75 months. Surgical time, blood loss, blood transfusion, and hospital length of stay of the patient was assessed. The workflow starts with patient undergoing a CT scan, which is used to generate a $3 \mathrm{D}$ model. Plastic surgeon and engineer meet for VSP which allows planning of osteotomies and reposition of bone based on age-appropriate normative model (generated from radiograph data) to achieve desired calvarial morphology. Templates are created at this point which are used intraoperatively to guide the initial placement of osteotomies and later arrangement of individual bone segments back on calvarium to achieve the normal morphology. Results showed mean surgical time was 326 min, mean estimated blood loss was $437.5 \mathrm{ml}$, mean blood transfusion was 329.25 $\mathrm{ml}$ and the mean hospital length of stay was 4.5 days.

Wilbrand, et al. [28] used 3-D photography to track the clinical course of correction of CS. There were 28 patients having a mean age of 7.8 months comprising of 19 males and 9 females. Outcomes such as CI, anterior symmetry ratio, posterior symmetry ratio, and anterior skull volume were analyzed. Photographic scans were obtained between 1 day and 2 weeks before surgery and at 10 days to 8 weeks after the surgery using Canfield VECTRA-360-four pod system and mirror software. The Cranioform Analytics 3.0 software was used to match pre and post-operative $3 \mathrm{D}$ photographs to analyze the parametric outcomes. The CI in Scaphocephaly changed from $75.1 \%$ to a median value of $77.4 \%$. The anterior symmetry ratio for coronal synostoses improved from 0.943 to 0.949 (a value of 1.0 represents perfect symmetry). The posterior symmetry ratio improved from 0.733 to 0.808 postoperatively in one single lambdoidal synostosis. In trigonocephaly, the median anterior skull volume rose from 528 to $601 \mathrm{ml}$.

Iyer, et al. [29] deployed CAD/CAM during reconstructive 
surgery for CS. Thirty two patients having a median age of 11 months underwent this study where 18 patients were male and 14 were female. Superior sagittal sinus (SSS) deviation, operative duration, blood loss, and hospital length of stay were evaluated. Technique described in the paper starts with producing digital models using preoperative imaging, followed by a VSP between surgeon and engineer. The session's outcome generates cutting guides that are used to mark osteotomies on the calvarium, and bone fragments are placed back via plate and screw system. Mean $( \pm$ SD) operative duration was $8.4 \mathrm{~h}( \pm 1.3)$. Median estimated blood loss was $350 \mathrm{~mL}$. Median hospital length of stay was 3.5 days. All patients tolerated surgical intervention without significant complications. Sagittal sinus deviation from the sagittal suture was maximal in unicoronal synostosis patients $(10.2 \pm 0.9 \mathrm{~mm})$.

García-Mato, et al. [30] used combination of CAD/CAM, 3D Printing, VSP, and intraoperative navigation to devise a workflow for CS surgery. The workflow was followed in 5 patients ( 2 male and 3 female) having a mean age of 11 months. Root-mean-squared error (RMSE), interfrontal angle, and transverse forehead width were evaluated to assess the accuracy of intraoperative registration. The proposed workflow starts with CT scan data acquisition which is used to generate a $3 \mathrm{D}$ model of the skull using a 3D Slicer software. After the model was smoothened and postprocessed, the output 3D model was used as a reference for computer assisted preoperative planning. CAD software (Freeform plus) was used for planning osteotomies, creating 3D models of patient-specific cutting guides, simulating affected bone remodeling, generating 3D models of templates, and generating 3D models of the skull. Following this, surgical guides and templates were 3D printed using SLS method and polyamide material (KLS Martin Group, Germany). For intraoperative navigation, CranioNav was developed to assist surgeons during the procedure including an optical tracking system called Polaris Spectra (NDI, Canada) for real-time tool position. Primary registration, which was performed using six landmarks on the 3D printed surgical guides, yielded an average RMSE of $0.94 \pm 0.27 \mathrm{~mm}$ for the five surgeries under study. The average time required to perform the registrations was $50 \pm 10$ seconds. Secondary registration, which was performed using the position of six resorbable pins attached intraoperatively, yielded an average RMSE of $1.30 \pm 0.47 \mathrm{~mm}$. The average duration of secondary registrations was $40 \pm 14$ seconds. The average error between target and postoperative interfrontal angles was $3.63^{\circ}$. The average error between target and transverse forehead width was $1.89 \mathrm{~mm}$.

Ganesh, et al. [31] exhibited the use of CAD/CAM, and VSP in treatment of CS using CVR. A total of 10 patients were divided into two groups; 5 Virtual surgical planning group (VFOU) having a mean age of 7.4 months and 5 Non-template Fronto-Orbital Unit (NFOU) group which was the control group having a mean age of 7 months. Time of surgery, blood loss, and length of hospital stay was considered while comparing the two groups. The VFOU group followed a workflow which was initiated by acquisition of CT images, followed by its conversion to DICOM file format. The next step involved virtual rendition of cranial construct using Osteo3D software which was the basis for VSP teleconference between surgeon and engineer. The outcomes of the VSP result in fabrication of the template/ positioning guide using CAD/CAM. The Mean $( \pm$ SD) time of surgery was $364 \pm 32.09$ minutes in NFOU group when compared to the VFOU group where the Mean ( \pm SD) time of surgery was $310 \pm 29.15$ minutes. The Time of surgery was significantly low in the VFOU group than the NFOU Group with $\mathrm{p}=0.021(<0.05)$. Intra-operative mean blood loss was found to be $250 \pm 23.45 \mathrm{ml}$ in NFOU group compared to $218 \pm 13.03 \mathrm{ml}$ in the VFOU group. There was a statistically significant difference in p value $=0.014(<0.05)$ between the groups. There was no statistically significant difference in the length of hospital stay with an average of 5 days in both the groups.

Andrew, et al. [32] used VSP to improve the surgical outcomes for the CS repair. For this study, a total of 63 patients were divided into 2 groups; 35 non-VSP/control (mean age $=27.23$ months) and $28 \mathrm{VSP}$ (mean age $=19.75$ months) patients. Number of screws, number of plates, blood loss, hospital stay, postoperative complications, and operative time was compared amongst the groups. The VSP group's computer-assisted surgical planning begins with CT scan acquisition. These scans are forwarded to 3D Systems (Littleton, Colo) for 3D construction and virtual surgery. Following step involves a teleconference between the craniofacial surgery and biomedical engineering team, resulting in generation of complete $3 \mathrm{D}$ images in which virtual osteotomies and bone flap manipulations are performed until the optimal shape and cranial index for the individual are obtained. The cutting/trimming aids and final positioning guides are generated based on the VSP. 100 screws or rivets were used in the VSP group in comparison to 66 screws (no rivets) in the surgical controls $(\mathrm{p}<0.01)$. No difference in the number of plates opened from original packaging was found (4.89, controls and 4.39, VSP). The Intraoperative blood loss was comparable in both groups, $172.21 \mathrm{ml}$ and $182.86 \mathrm{ml}$ for controls and VSP cases, respectively. VSP cases stayed in hospital significantly longer than control patients, 5.39 days versus 3.97 days $(p<$ 0.01 ), respectively. Postoperative complications were similar in both groups. The operative time for all procedures was on average 321.44 minutes in the control group and 265.61 in the VSP cases $(\mathrm{p}=0.01)$.

Khechoyan, et al. [33] utilized CAD/CAM assisted prefabricated templates to prove its merit in CS reconstruction. Thirty seven patients comprising of 20 male and 17 females were involved in the study, which were divided into 2 groups; 14 template (mean age $=11.5$ months) and 23 no template/control (mean age $=10.2$ months) patients. Area under the curve (AUC), duration of operation, blood loss, blood transfusion and length of hospital stay were considered for outcomes. The template group used CAD software in conjunction with normative data to manufacture patient-specific stainless steel bandeau templates. Estimated blood loss, volume of blood product transfusion and length of hospital stay was not statistically different between the two groups. The duration of operation was significantly shorter for the template group (212 vs. $258 \mathrm{~min}, \mathrm{p}<0.001)$. At the postoperative time point, the mean AUC for the template and the no-template groups was significantly different (199 vs. $309 \mathrm{~mm}^{2}$, p < 0.05). Mean reduction in AUC at the time of operation was $74 \%$ for the template group and $56 \%$ for the no-template (control) group, respectively $(\mathrm{p}<0.05)$. The mean absolute reduction in AUC was also significantly different between the template and no-template, control groups (663 vs. $\left.500 \mathrm{~mm}^{2}, \mathrm{p}<0.001\right)$. 
Freudlsperger, et al. [34] utilized 3D photogrammetry as a tool to improve the CS surgery. Eighteen patients that were all males, had a mean age of 9 months at the time of the surgery. Intracranial volume (total, frontal and posterior) was assessed in the study. Photographic scans were performed using a Canfield VECTRA-360-four pod system (Canfield science, Fairfield, NJ, USA) to generate 3D data. The 3D data was analyzed using Cranioform Analytics 4.0 software (Cranioform, Alpnach, Switzerland). Pre- and postoperative photogrammetric data was compared using Geomagic Studio 6.0 (Raindrop Inc., Durham, NC, USA) to visualize the areas of skull where intracranial volume was increased or decreased. The measurement of the mean pre- and post-operative intracranial volume revealed a significant increase of the frontal volume $(707.5 \mathrm{ml}$ vs. $833.3 \mathrm{ml})(\mathrm{p}=0.0054)$ after fronto-orbital advancement. The mean posterior volume's increase was less pronounced $(654.5 \mathrm{ml}$ vs. $686.0 \mathrm{ml})(\mathrm{p}=0.3376)$. The operative procedure increased the mean total volume $(1362.0 \mathrm{ml}$ vs. $1519.3 \mathrm{ml})$ in a significant manner $(\mathrm{p}<0.01)$. Pre-operatively, the mean frontal volume of children with metopic synostosis was significantly lower compared with the control group $(654.5$ $\mathrm{ml}$ vs. $911.0 \mathrm{ml})(\mathrm{p}<0.001)$. Consequently, mean total volume was significantly decreased $(1362.0 \mathrm{ml}$ vs. $1541.8 \mathrm{ml})(\mathrm{p}=$ 0.0031 ). Posterior volume did not differ from the control group in a significant manner $(654.5 \mathrm{ml}$ vs. $630.8 \mathrm{ml})(\mathrm{p}=$ 0.4322 ). Post-operatively, no significant difference in the frontal, posterior and total volume was present, indicating a considerable increase in the intracranial volume especially in the frontal area due to the fronto-orbital advancement.

\section{Discussion}

The results of the review were conclusive. All studies [23-34] reported advancements in surgical outcomes by introducing technologies that can make the surgical process more accurate, efficient and reliable. Figure 4 provides a breakdown on usage of various technologies deployed as per the findings of this review.

\section{$\mathrm{CAD} / \mathrm{CAM}$}

Computer-aided design and manufacturing can be utilized in planning of osteotomies and placement of bone segments which allows to achieve a standardized, objective and precise correction of CS, eliminating any guesswork or subjectivity from the surgery to achieve more

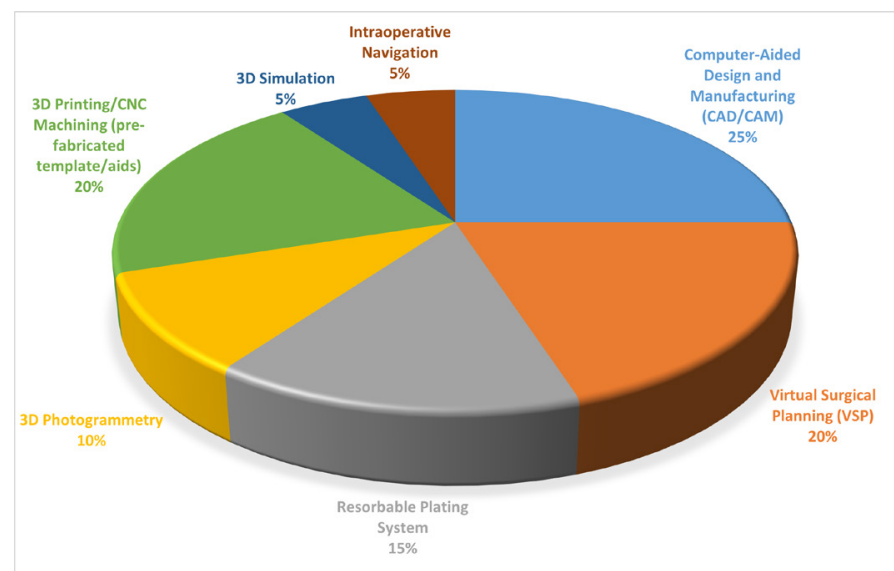

Figure 4. Technology share based on the findings of the review reproducible surgical results [27,33]. CAD/CAM technology can potentially localize dural venous sinuses during CS reconstructive surgery, which diminishes the risk of sinus injury by allowing surgeons to appropriately plan burr holes and cuts, increasing surgeon's confidence from enhanced safety of the procedure and minimize patient morbidity [29]. When included in workflow for patient specific correction of CS, CAD/CAM can be used for planning surgical osteotomies, generating patient specific 3D models, and cutting guides to assist during the surgery [30]. Ganesh, et al. compared the template group (TFOU) with the nontemplate group where $\mathrm{CAD} / \mathrm{CAM}$ was used to prefabricate template/positing guide, enhancing the visualization of the surgical problem with a multidimensional perspective of the deformity [31].

\section{VSP}

Virtually Surgery Planning allows reproducible objective results that improve outcomes regardless of the expertise of the surgeon and may be most useful for complex multisuture CS where freehand osteotomy and shaping of the calvarium may result in less predictable result. In addition, patients and families have a significantly better understanding due to the preoperative counselling and postoperative rehabilitation, which results in better alignment of hopes and expectations between families and surgeons [27]. VSP provides surgeons with clear 3D visualization of a cranial dysmorphology and helps develop an efficient surgical plan via formulation of intra-operative goal that can be achieved with precision [31]. It can also help inexperienced surgeons and residents as an educational tool to help navigate and understand the scope of the surgery. Operative time was reduced by 45 minutes in single suture patients and 101 minutes in multi suture patients using VSP when compared with non-VSP group [32]. This is due to the fact that VSP shifts part of the surgery to a much controlled and calculative pre-surgical environment where both the engineering as well as medical teams are working in tandem to achieve the same goal.

\section{$3 \mathrm{D}$ printing/CNC machining}

Surgical templates/aids can be manufactured via 3D printing or CNC machining depending upon their application, which leads to reliable outcomes, increased efficiency in the operating room and decreased operative times due to elimination of any guesswork [27,31]. The duration of operation was significantly shorter for the template group that used patient-specific stainless steel bandeau template compared to the non-template group (212 vs. $258 \mathrm{~min}, \mathrm{p}<0.001)$, that also led to a greater reduction in AUC ( $74 \%$ for the template group and $56 \%$ for the notemplate group, $p=0.016$ ) [33]. García-Mato, et al. proposed a two-step registration procedure using $3 \mathrm{D}$ printed surgical guides that enables the user to repeat the registration multiple times during the surgery minimizing navigation error and also suggested using a 3D printed normative skull model as a reference during virtual planning [30]. A wide variety of materials can be manufactured between the two technologies ranging from biocompatible plastics to tougher materials like stainless steel and titanium. Since medical VR and image processing software can export "STL" file formats, it requires none to some manipulations in $3 \mathrm{D}$ Printing and CNC Milling, respectively to manufacture 
cutting/marking/position guides and templates for intraoperative use.

\section{Resorbable plating system}

Ultrasound-assisted pinned resorbable plating system provides timesaving and stable osteosynthesis without increase in operation time or any signs of foreign body reaction [24]. Placement of resorbable plates on the calvarial bone is safe, stable, results in satisfactory aesthetic improvement, and does not hamper the ossification of the cranial bones in the frontal area [25]. Also, the learning curve of using the plating system improves over time, leading to reduction in length of surgery since surgeons become familiar with their application and correct usage. The use of resorbable plating system for CVR is safe, reproducible, and inexpensive modality with very low complication rates, while having 3D stability, rigid fixation without causing growth restriction, and lower likelihood of need for removal [26].

\section{$3 \mathrm{D}$ photogrammetry}

3D photogrammetry/photography is safe, fast and simple, requires no sedation and is independent of the examiner [28] which can be translated to minimizing error. It can be substituted in place of a routine pre- and post-operative CT scan [34], avoiding radiation in small infants which can lead to malignancies in later life [35], resulting in decreased patient morbidity.

\section{D simulation}

Computer-aided simulated operation will play an important role in making surgical plans as it not only reduces operative time, but also decreases intraoperative bleeding loss, thus reducing the rate of blood transfusion; besides it also gives doctors a good surgical experience and an understanding of operation procedure [23].

\section{Intraoperative navigation}

Registration required for intraoperative navigation does not increase surgical time and has a short learning curve that enables an accurate translation of preoperative surgical planning into the operating room, thus improving the reproducibility of CS surgeries and reducing inter-surgeon variability [30].

\section{Conclusion}

The field of industrial engineering in medical sciences has seen massive growth over the past decade and continues to grow in the year $2021[38,39]$, especially when latest technologies like VR/AR and CAD/CAM is utilized with VSP [36,37,40], which facilitates the production of guides/ aids for intra-operative use via $3 \mathrm{D}$ printing/AM, and $\mathrm{CNC}$ machining. For CS repair, it has been shown that computeraided pre-surgical planning can provide multiple advantages, including better visualization of the abnormality in 3D space [31] and shorter operative times [32]. Thus, there is a need to develop a workflow for the surgical treatment of CS using VSP, CAD/CAM, VR/AR, 3D printing and CNC machining to help surgeons achieve predictable and reproducible results in the operating room. Though the knowledge and skill of a surgeon is unparalleled, these technologies can definitely be an armamentarium, helping them achieve even better surgical outcomes. A standardized workflow will result in improving the speed (operative time) by moving part of the surgery to a more controlled pre-surgical planning environment and accuracy (precision) by achieving intricate and precise cuts on the calvarial bone, resulting in fewer errors during the surgery. This will also help younger/less experienced surgeons better visualize and rehearse the treatment to address any concerns regarding the nature of the surgery. Additionally, the surgical planning outcomes can be shared with patients' family, leading to an increased level of understanding and trust between the physician and the parents.

\section{Acknowledgment}

We gratefully thank Dr. S. Anthony Wolfe for sharing archive images and research articles.

\section{References}

1. Boulet SL, Rasmussen SA, Honein MA. A population-based study of craniosynostosis in metropolitan Atlanta, 1989-2003. Am J Med Genet A. 2008;146A(8):984-991.

2. Johnson, D, Wilkie A. Craniosynostosis. Eur J Hum Genet. 2011;19:369-376.

3. Wilkie AOM, Johnson D, Wall SA. Clinical genetics of craniosynostosis. Curr Opin Pediatr. 2017;29(6):622-628.

4. Kung TA, Vercler CJ, Muraszko KM, Buchman SR. Endoscopic Strip Craniectomy for Craniosynostosis: Do We Really Understand the Indications, Outcomes, and Risks?. J Craniofac Surg. 2016;27(2):293-298.

5. Delashaw JB, Persing JA, Broaddus WC, Jane JA. Cranial vault growth in craniosynostosis. J Neurosurg. 1989;70(2):159-165.

6. Clayman MA, Murad GJ, Steele MH, Seagle MB, Pincus DW. History of craniosynostosis surgery and the evolution of minimally invasive endoscopic techniques: the University of Florida experience. Ann Plast Surg. 2007;58(3):285-287.

7. Tessier P, Guiot G, Rougerie J, Delbet JP, Pastoriza J. Ostéotomies cranio-naso-orbito-faciales. Hypertélorisme [Cranio-nasoorbito-facial osteotomies. Hypertelorism]. Ann Chir Plast. 1967;12(2):103-118.

8. Czerwinski M, Hopper RA, Gruss J, Fearon JA. Major morbidity and mortality rates in craniofacial surgery: an analysis of 8101 major procedures. Plast Reconstr Surg. 2010;126(1):181-186.

9. Nguyen C, Hernandez-Boussard T, Khosla RK, Curtin CM. A national study on craniosynostosis surgical repair. Cleft Palate Craniofac J. 2013;50(5):555-560.

10. Lee HQ, Hutson JM, Wray AC, et al. Analysis of morbidity and mortality in surgical management of craniosynostosis. J Craniofac Surg. 2012;23(5):1256-1261.

11. Seruya M, Sauerhammer TM, Basci D, et al. Analysis of Routine Intensive Care Unit Admission following Fronto-Orbital Advancement for Craniosynostosis. Plastic and Reconstructive Surgery. 2013;131(4):582e-588e.

12. Goobie SM, Zurakowski D, Proctor MR, et al. Predictors of clinically significant postoperative events after open craniosynostosis surgery. Anesthesiology. 2015;122(5):10211032

13. Oppenheimer AJ, Ranganathan K, Levi B, et al. Minimizing transfusions in primary cranial vault remodeling: the role of aminocaproic acid. J Craniofac Surg. 2014;25(1):82-86.

14. Ing C, Hegarty MK, Perkins JW, et al. Duration of general anaesthetic exposure in early childhood and long-term language and cognitive ability. Br J Anaesth. 2017;119(3):532-540.

15. Maruthappu M, Duclos A, Lipsitz SR, Orgill D, Carty MJ. Surgical learning curves and operative efficiency: a cross- 
specialty observational study. BMJ Open. 2015;5(3):e006679.

16. Faberowski LW, Black S, Mickle JP. Blood loss and transfusion practice in the perioperative management of craniosynostosis repair. J Neurosurg Anesthesiol. 1999;11(3):167-172.

17. Jimenez DF, Barone CM. Intraoperative autologous blood transfusion in the surgical correction of craniosynostosis. Neurosurgery. 1995;37(6):1075-1079.

18. Kearney RA, Rosales JK, Howes WJ. Craniosynostosis: an assessment of blood loss and transfusion practices. Can J Anaesth. 1989;36(4):473-477.

19. Ririe DG, David LR, Glazier SS, Smith TE, Argenta LC. Surgical advancement influences perioperative care: a comparison of two surgical techniques for sagittal craniosynostosis repair. Anesth Analg. 2003;97(3):699-703.

20. Tunçbilek G, Vargel I, Erdem A, Mavili ME, Benli K, Erk Y. Blood loss and transfusion rates during repair of craniofacial deformities. J Craniofac Surg. 2005;16(1):59-62.

21. Hammoudeh JA, Howell LK, Boutros S, Scott MA, Urata MM. Current Status of Surgical Planning for Orthognathic Surgery: Traditional Methods versus 3D Surgical Planning. Plast Reconstr Surg Glob Open. 2015;3(2):e307.

22. Moher D, Liberati A, Tetzlaff J, Altman DG; PRISMA Group. Preferred reporting items for systematic reviews and metaanalyses: the PRISMA statement. PLoS Med. 2009;6(7):e1000097.

23. Li X, Zhu W, He J, et al. Application of computer assisted threedimensional simulation operation and biomechanics analysis in the treatment of sagittal craniosynostosis. J Clin Neurosci. 2017;44:323-329.

24. Freudlsperger C, Castrillon-Oberndorfer G, Baechli H, Hoffmann J, Mertens C, Engel M. The value of ultrasoundassisted pinned resorbable osteosynthesis for cranial vault remodelling in craniosynostosis. J Craniomaxillofac Surg. 2014;42(5):503-507.

25. Salokorpi N, Sinikumpu JJ, Iber T, et al. Frontal cranial modeling using endocranial resorbable plate fixation in 27 consecutive plagiocephaly and trigonocephaly patients. Childs Nerv Syst. 2015;31(7):1121-1128.

26. Branch LG, Crantford C, Cunningham T, et al. Long-Term Outcomes of Pediatric Cranial Reconstruction Using Resorbable Plating Systems for the Treatment of Craniosynostosis. J Craniofac Surg. 2017;28(1):26-29.

27. Mardini S, Alsubaie S, Cayci C, Chim H, Wetjen N. Threedimensional preoperative virtual planning and template use for surgical correction of craniosynostosis. J Plast Reconstr Aesthet Surg. 2014;67(3):336-343.

28. Wilbrand JF, Szczukowski A, Blecher JC, et al. Objectification of cranial vault correction for craniosynostosis by threedimensional photography. J Craniomaxillofac Surg. 2012;40(8):726-730.

29. Iyer RR, Wu A, Macmillan A, et al. Use of computer-assisted design and manufacturing to localize dural venous sinuses during reconstructive surgery for craniosynostosis. Childs Nerv Syst. 2018;34(1):137-142.

30. García-Mato D, Ochandiano S, García-Sevilla $\mathrm{M}$, et al. Craniosynostosis surgery: workflow based on virtual surgical planning, intraoperative navigation and $3 \mathrm{D}$ printed patientspecific guides and templates. Sci Rep. 2019;9(1):17691.

31. Ganesh P, Mahipathy SRRV, Rajan VTT, et al. Traditional Versus Virtual Surgery Planning of the Fronto-Orbital Unit in Anterior Cranial Vault Remodeling Surgery. J Craniofac Surg. 2021;32(1):285-289.

32. Andrew TW, Baylan J, Mittermiller PA, et al. Virtual Surgical Planning Decreases Operative Time for Isolated Single Suture and Multi-suture Craniosynostosis Repair. Plast Reconstr Surg Glob Open. 2018;6(12):e2038.

33. Khechoyan DY, Saber NR, Burge J, et al. Surgical outcomes in craniosynostosis reconstruction: the use of prefabricated templates in cranial vault remodelling. J Plast Reconstr Aesthet Surg. 2014;67(1):9-16.

34. Freudlsperger C, Steinmacher S, Bächli H, Somlo E, Hoffmann J, Engel M. Metopic synostosis: Measuring intracranial volume change following fronto-orbital advancement using threedimensional photogrammetry. J Craniomaxillofac Surg. 2015;43(5):593-598.

35. Brenner D, Elliston C, Hall E, Berdon W. Estimated risks of radiation-induced fatal cancer from pediatric CT. AJR Am J Roentgenol. 2001;176(2):289-296.

36. Chim H, Wetjen N, Mardini S. Virtual surgical planning in craniofacial surgery. Semin Plast Surg. 2014;28(3):150-158.

37. Kim Y, Kim H, Kim YO. Virtual Reality and Augmented Reality in Plastic Surgery: A Review. Arch Plast Surg. 2017;44(3):179187.

38. Chae MP, Rozen WM, McMenamin PG, Findlay MW, Spychal RT, Hunter-Smith DJ. Emerging applications of bedside 3D printing in plastic surgery. Frontiers in Surgery. 2015;2:25.

39. Kimberly Mlitz (2021). https://www.statista.com/ statistics/796237/worldwide-forecast-growth-3d-printingmarket/

40. Zhao L, Patel PK, Cohen M. Application of virtual surgical planning with computer assisted design and manufacturing technology to cranio-maxillofacial surgery. Arch Plast Surg. 2012;39(4):309-316. 